\title{
Parchment Paper
}
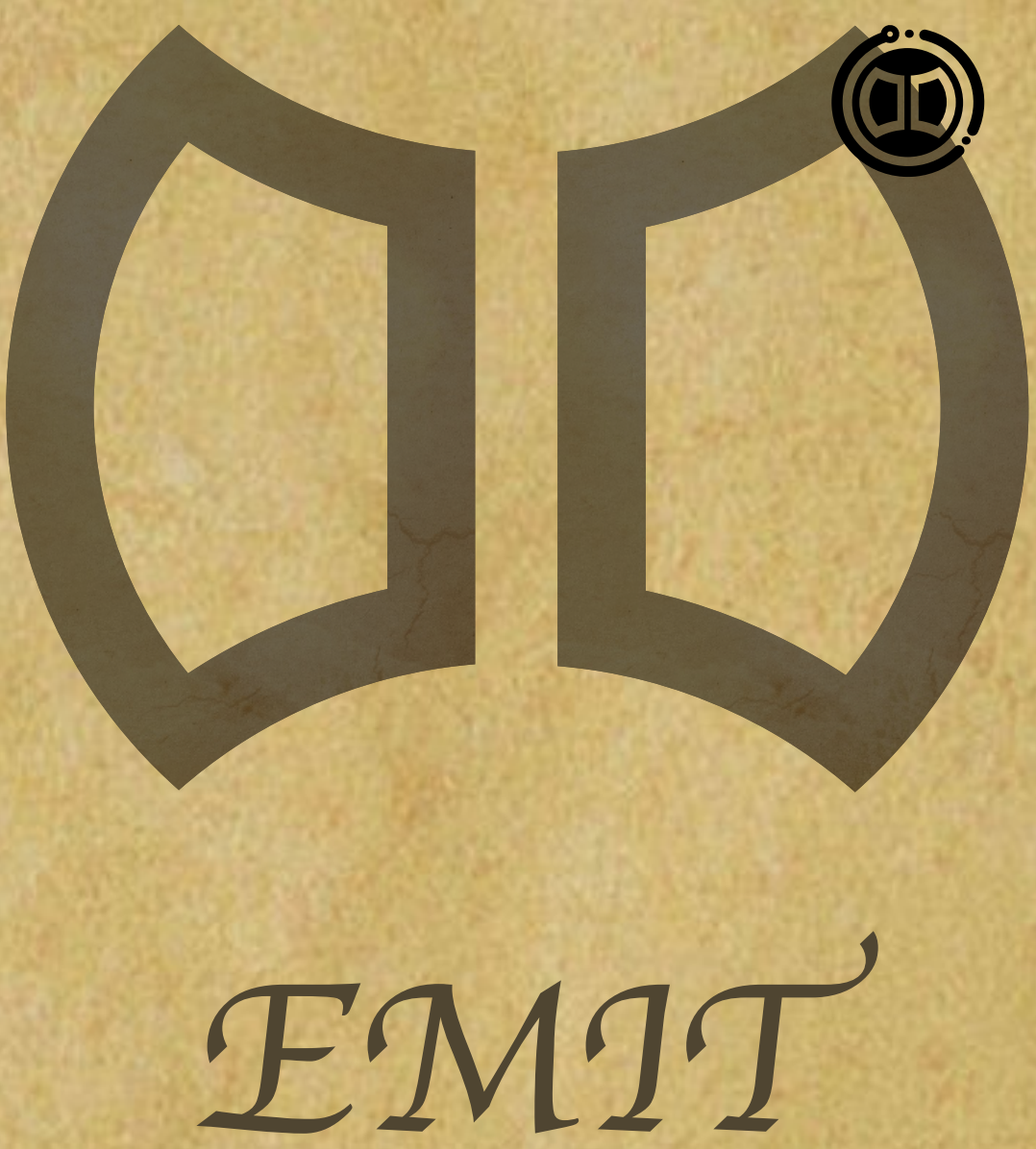

Constructing wealth with technology

$v_{3}$ 


\section{Table}

7. Abstract

21. Definitions

Parchment Paper

Decentralized World (DW)

Decentralized World Economy (DeWe)

Blockchain Industry

212. What is $\mathcal{E M I T}$

3.1. $\mathcal{E M T} \mathcal{T}$ Decentralized World

3.2. Electronical Material Information Technology

A. Technological Conditions

B. Social Conditions

3.3. EMIT's Three Major Systems

A. EMIT Core Network

B. $\mathcal{E P O C H}$ Ecosystem

C. GDP Financial Market

2V. Why $\mathcal{E} \mathcal{M} \mathcal{T}$ is $\mathcal{N}$ eeded

4.1. Limitations

A. Universal equivalent and asset classification

B. Internal Limitations

C. External Limitations 
4.2. Break through limitations

$\begin{array}{lr}\text { A. Infrastructure Upgrades } & 17\end{array}$

B. Upgrading of Economic Behavior Diversity: $\quad 17$

4.3. The Role of $\mathcal{E M I T} \quad 18$

A. EMIT' Core Network 19

B. $\mathcal{E P O C H}$ Ecological Cluster $\quad 19$

$\begin{array}{ll}\text { C. GDP Financial Marketplace } & 20\end{array}$

$\begin{array}{ll}\text { V. EMIT's Plan } & 21\end{array}$

5.1. Prelude (2 Months) 21

A. World Tree (January 2021) 21

B. Birth of Pioneers (February 2021) 21

5.2. Pioneers (1 Year) 21

$\begin{array}{ll}\text { A. Start (March 2021) } & 21\end{array}$

$\begin{array}{ll}\text { B. Clan } & 21\end{array}$

C. Ark 22

5.3. Nation (3 Years) 22

5.4. World (5 Years) 22

VI. $\mathcal{E M I T}$ foundation $\quad 23$

VII. Participation in $\mathcal{E M I T} \quad 24$ 


\section{EMIT'Parchment Paper}

\section{A Gstract}

$t$ the beginning of this paper, the decentralized world (DW) and decentralized world
economy (DeWe) were defined. And the blockchain industry was pointed out to be at the
initial stage of the decentralized world parallel to the real world. Then a set of systematic solutions, named Electronical Material Information Technology (EMIT), was proposed, after which the development direction and path of the decentralized world economy was put forward, from its limitations, by arguing how to provide sufficient and necessary basic conditions for the decentralized world development. In the end, the $\mathcal{E M I T}$ ' was proved to be an effective reference for building the decentralized world from the basic level to the application level and enabling its sustainable development.

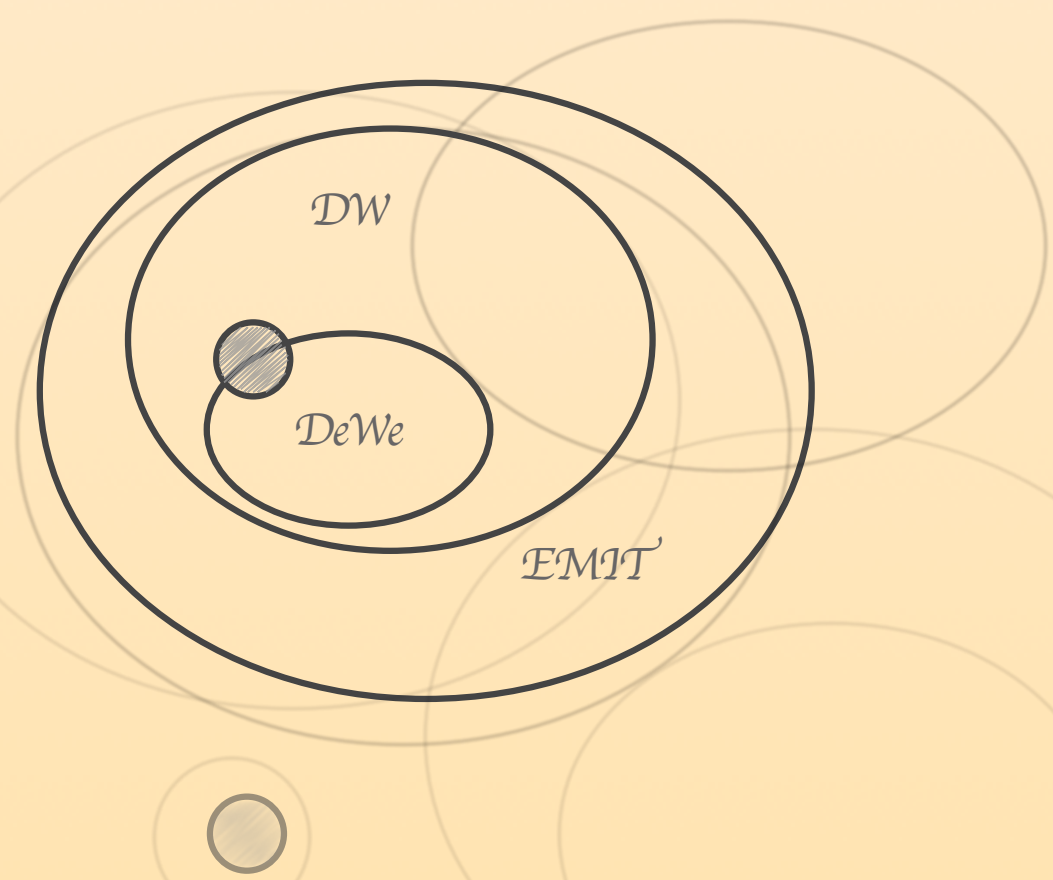




\section{Definitions}

\section{Parchment Paper}

Unlike a white paper that sets out the technical details of a product, a parchment paper focuses on promoting ideas. It presents an analysis of the current situation and problems as well as corresponding constructive advice.

\section{Decentralized World (DW)}

The decentralized world, as defined by $\mathcal{E M I T}$, is an objective existence that exists in a generalized cyberspace, with real humans as economic agents, as an external extension of the real world economy. The decentralized world has self-consistent economic rules, and can generate a larger and more complicated economic structure than that of the real world.

The decentralized world is not a game world as currently recognized in society. Humans engage in the decentralized world as the economic subjects, not the game characters. As the economic subjects, humans will carry out various real activities in the decentralized world, in which their commercial and trading Gehavior remain an important foundation. Moreover, the virtual word develop and improve into a new political, economic and social space, supported by the new generation of information technology. Once it has been created, it will never disappear.

\section{Decentralized World Economy (DeWe)}

The sum of the creation, realization, transformation and structural relations of value and wealth covered by the decentralized world is part of the overall human economy and an outgrowth extension of the real world economy.

\section{Blockchain Industry}


It is believed in $\mathcal{E M} 2 \mathcal{T}$ that, in the context of the development and gradual popularization of systems such as $\mathcal{D} e \mathcal{F} i$, N $\mathcal{F} \mathcal{T}$ ' applications, cross-chain technology, high $\mathcal{T}$ 'PS and complex decentralized applications in the current era, the value and relationship system constructed by the blockchain industry is already gradually forming the prototype of the decentralized world economy. 


\section{What is $\mathcal{E M T \mathcal { T }}$}

ssentially, the meaning of $\mathcal{E M} \mathcal{T} \mathcal{T}$ ' can be explained from three levels:

- A complete decentralized world (EMIT'Decentralized World)

- The technology underlying the construction of these projects (EMIT)

- A collection of projects that propel the birth and development of this decentralized world (the three $\mathcal{E} M \mathcal{M} \mathcal{T}$ systems)

\section{1. $\mathcal{E} M \mathcal{T} \mathcal{T}$ Decentralized World}

As a complete decentralized world, the material base of $\mathcal{E M} \mathcal{T} \mathcal{T}$ ' is a contiouously developing information system on three different levels constructed by "Electronical Material Information Technology". In the EMIT's decentralized world, the economic structure and cycle transcend those in the real world.

\subsection{Electronical Material Information Technology}

$\mathcal{E M I T}$ is an acronym for Electronical-Matter-Information-Technology, a new technical protocol and reference implementatio for the construction of the decentralized world based on electronic information technology. $\mathcal{E M I T}$ depends on two basic conditions: technical conditions and social conditions.

\section{A. Technological Conditions}

Technologically, $\mathbb{E M} 2 \mathcal{T}$ is a new emerged concept, but it contains some key technologies from the web2.o era until now, as well as innovations and converges on these technologies, including some key technological innovations in centralized information technology and decentralized information technology. 
Those in decentralized information technology include but are not limited to: consensus algorithms with high $\mathcal{T}$ 'PS and scalability, smart contracts supporting high complexity requirements, cross-chain technology, complex virtual asset protocols, $\mathcal{P}_{2} \mathcal{P}$ communication and privacy algorithms.

Those in centralized information technology include what people commonly see: cloud computing, cloud storage, artificial intelligence, We62.o, $5 \mathrm{G}$ and Internet of Things, etc.

\section{B. Social Conditions}

With the blockchain industry development in the past over ten years since the birth of Bitcoin $(\mathcal{B T} C)$ in 2009, thousands of virtual assets recognized by the community have been created. And a large amount of social capital has been invested into the prototype of the decentralized world where these assets are in.

Based on these technologies and diversified blockchain encrypted assets, a decentralized world can be constructed, which includes virtual things and operation rules. Some of them may have some extent reflect things and relationships in the real world.

However, unlike the popular cyberspace, it has similar properties to the real world, including objective existence, irrevocability, endogenous cycles, infinite evolvability and self-organization, high complexity, consensus decision-making and parallel operation.

People in reality can engage in such an infinite and vibrant decentralized world by operating on the interfaces of electronic devices, and then conduct creation, production, exchange and interaction. The decentralized world, by relying on the indirect economic connections with the real world and its self-generated economic cycle, can evolve on its own and form self-consistent and complicated economies and ecologies.

Eventually, the decentralized world will intersect with the real world at several points, serving as an outgrowth of the real world's economic cycle and helping to increase the world's total wealth. 


\title{
3.3. EMIT's Three Major Systems
}

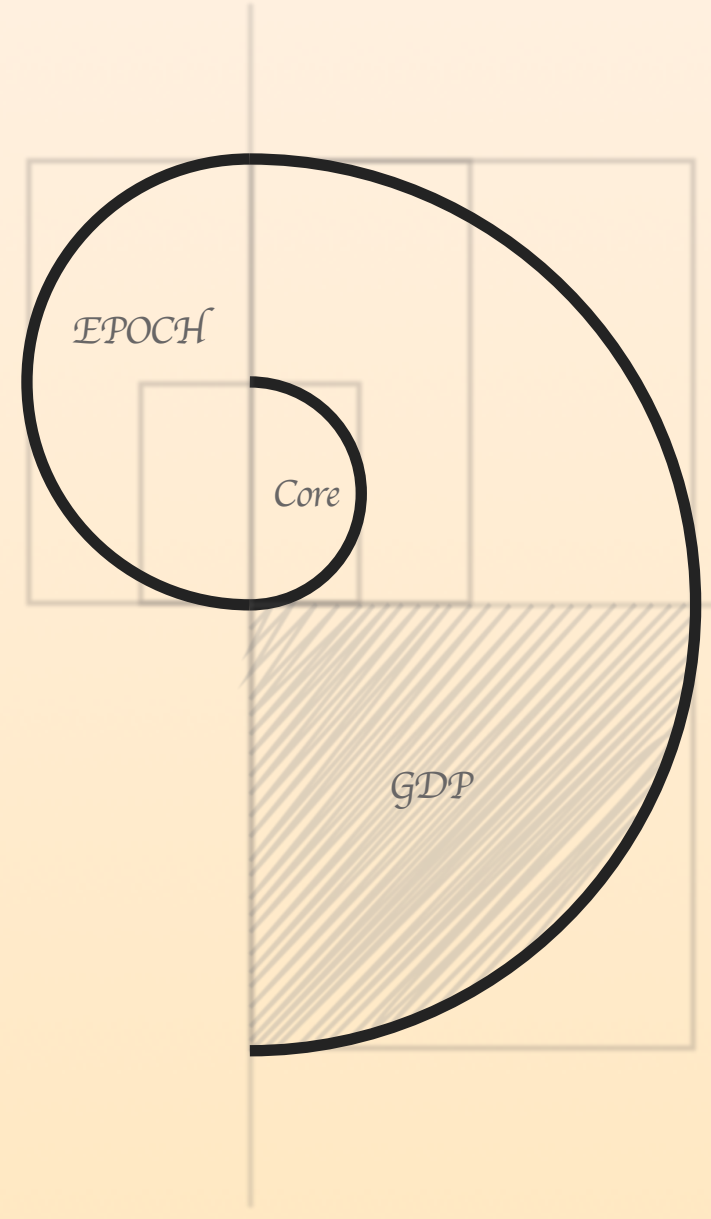

\author{
There are three major systems in the \\ architecture of $\mathcal{E M I T} \mathcal{T}$, namely, "IFMIT' Core \\ Network", "EPOCH Ecosystem" and "GDP \\ financial market", one of which is the basis for \\ the other.
}

\section{A. $\mathcal{E M I T}$ Core Network}

The EMIT' Core Network is a consensus network comprised of decentralized nodes, and it is the infrastructure that supports the operation of the entire $\mathcal{E M I T}$ 'system. It has the characteristics of the cutting-edge blockchain network, including: consensus protocols that naturally support sharding and cross-chain, infinitely scalable T'PS, convenience in developing complex decentralized applications, low-cost application deployment and operation, supporting complex asset structure, privacy protection and confidential transactions, and no native fungible tokens.

\section{B. $\mathcal{E P O C \mathcal { H }}$ Ecosystem}

The EPOCH ecosystem refers to a variety of decentralized ecologies running on the $\mathcal{E M I T}$ network based on the EPOCH protocol and framework, which is used to build the endogenous cycle of the decentralized world economy. The EMIT' Foundation will preset and operate a series of initial logic and evolve with the community, which will conduct various associated ecological $\mathcal{R} \& \mathcal{D}$ and consensus diffusion. In this way, combined with the cross-chain technology of the EMIT protocol, the whole decentralized world will be used by $\mathcal{E P O C H}$ as the development foundation of self-organization and self-evolution, expanding into diversified virtual entities and 
evolving into a more advanced ecosystem, ultimately resulting in a large amount of endogenous GDP.

\section{GDP Financial Market}

The GDP financial market is one of the most important core applications in $\mathcal{E P O C H}$, and a decentralized exchange and auction center for diverse assets. It supports diversified assets and trading behaviors.

These diversified assets, including fungible and non-fungible tokens, can be classified in terms of application as virtual things with simple or complex structures such as the universal equivalents, entities, materials, tools, organizations, locations, or virtual things that can be reflected in the real world.

Diversified behaviors include a rang of behaviors such as production, exchange, rent, lending, pledge, pre-purchase, option, cooperative business, merger and acquisition, combination of things and storage, etc.

This paper remains a parchment paper, aiming at explaining why $\mathcal{E M I T}$ is needed and what EMIT is in general, please refer to the EMIT White Paper for technical details. 


\section{Why EMIT is $\mathcal{N}$ eeded}

$\mathcal{T}$

he most important reason for the birth of $\mathcal{E M I T}$ is to tackle some limitations of the DeWe. The existence of these limitations has, to some extent, led to the single economic structure in the current blockchain industry and the difficulty in forming a stable support for the value of virtual assets.

\subsection{Limitations}

At present, the limitations of the DeWe can be divided into internal and external factors. The former refers to the inadequate diversification of assets and trading behaviors, while the latter the conflict between free will and traditional habitual behaviors and forces.

\section{A. Universal equivalent and asset classification}

Before explaining factors that lead to the limitations mentioned above, two concepts should be additionally mentioned: "universal equivalent" and "asset classification". They can separately reflect the current situation of the economic development in the decentralized world.

\section{Universal equivalent}

In terms of general economic concepts, the universal equivalent refers to a special commodity, which is separated from common commodities and represents the unified value of other commodities. For example, shells were used in the early history of human beings, gold and silver were used later, and the fiat money is used in modern society. As the universal equivalent in the decentralized world, fungible virtual assets with strong consensus have unique advantages. However, in reality, virtual assets, as new and risk assets, have strong volatility of fiat currency for fiat currency values due to real-world influences. Therefore, the only cryptocurrency that acts as the universal equivalent of the decentralized world in the entire decentralized world economy is the USDT', which is issued by the centralized. This can easily be seen from CoinMarketCap's 24- 
hour trading volume statistics, as the transaction volume of USDT accounts for almost half of that of the whole virtual market.

From the statistics of the issue of USDT from 2017 to 2020, it can be seen that it has been increasing since January 2020. The amount has increased by nine times. However, in the secondary cryptocurrency market, the exchange rate of USD $\mathcal{T}$ against USD fiat always remains stable, indicating that the total circulation market value of the cryptocurrency market in the decentralized world economy has exponentially increased since January 2020.

And coincidentally, it was from then that another field, $\mathcal{D} e \mathcal{F} i$, was strongly supported and developed after a long incubation period. In the next part, we will classify the assets in the decentralized world, and $\mathcal{D} e \mathcal{F} i$ is one of the areas where very important circulating assets are attributed.

\section{Asset Classification}

On CoinMarketCap: there are 7,793 kinds of recorded virtual currencies at present, and 3,889 with data records. All of them are fungible assets. Except for some community airdrops and other activities, a price must be paid to acquire valuable assets in general, and this act of paying a price to get an asset has important implications for the decentralized world. In terms of the original repayment purpose of assets, the currently meaningful virtual assets can be classified into categories listed below:

Interface assets: $\mathcal{A}$ n asset that acts as a bridge between the decentralized world and the real world. Generally, they are centrally issued, and used as a medium asset to directly map real world assets to decentralized world assets, such as USDT, USDC and some other tokens. There are also decentralized issuances, such as $\mathcal{D} A \mathcal{A}$, but the relative liquidity of such assets is clearly insufficient.

Genesis Assets: Used as an asset to maintain and stabilize the decentralized world, it is issued by decentralization and is generally a native token of a certain blockchain network. The pursuit of the value of such assets can maintain and stabilize the decentralized world. Generally, 
to acquire such assets, one needs to provide a large amount of negentropy (POW-consensus coins) or give up the current value of the token (POS-consensus coins) to accomplish the task of normalizing and safeguarding the decentralized network (stabilizing the decentralized world), and receive the reward of that token. Examples of tokens are $\mathcal{B} \mathcal{T}^{\prime} \mathcal{C}, \mathcal{E} \mathcal{T} \mathfrak{H}, \mathcal{E} O S$, etc.

Liquid assets: Another kind of application emerged in the decentralization field after the rise of Defi, aiming at solving the liquidity problem of virtual assets. Pledging assets with unstable value and insufficient liquidity can acquire other assets with more stable value and circulation, or several certain assets can be directly used as the liquidity guarantee of free trade in the market. In these processes, liquidity providers reap a virtual asset as a reward for the price they pay to facilitate liquidity, and this asset is the liquid asset.

Application assets: These assets make up the majority of the CoinMarketCap list, and are generally purchased by users who need to be paid for certain services. Examples include the large number of $\mathcal{E R C} 20$ tokens and gaming tokens on $\mathcal{E}$ thereum. Such assets are of mixed quality and are highly speculative. And often the ones that survive are related to read business.

Organizational assets: Such assets are generally acquired due to effective social organization structures formed by user groups. Driven by such assets, users form organized social groups to accomplish the organizer's goals directly or indirectly. Because of the structure and issue growability of virtual assets, such organizations are generally similar to pyramidal structures. And many existing ones are Ponzi organizations.

In fact, many virtual assets have different characteristics at the same time, and it is sometimes difficult to distinguish them.

\section{B. Internal Limitations}

From the above discussion, it can be seen that, in the decentralized world, the cryptocurrency market has been growing, and the diversity of asset classification is not insufficient. But through careful observation, it is found that the monolithic of transaction behaviors and purposes has led 
to an over-concentration of trading volume on the head few assets. Due to the convenience in issuing virtual assets, the homogenization of Gehaviors remains serious.

\section{Insufficient diversification of economic behaviors}

In the current decentralized world, most behaviors related to virtual assets are financial behaviors, such as recharging, withdrawing, buying and selling, pledging, lending and arbitrage. And, the production behaviors all take place in the real world. Looking back on the real world, it can be found that although its financial system is very complex and becomes more abstract at the top levels, the financial industry in the real world ultimately needs to serve the real economy and propel the development of the three main industries after the optimizing allocation of capital via the market. In the current decentralized world, productive behavior is non-existent, all kinds of financial behavior allocated funds, what exactly is allocated, who has a strong speculative ability?

In addition, the basis of real-world finance is trust, and in the current decentralized world, the support base for this trust is relatively weak. Therefore, all financial behaviors are pledging existing assets to acquire another type of asset, and it must be an over pledge.

\section{Insufficient iversification of assets}

As discussed previously, assets are categorized based on original repayment purposes, so the lack of behavioral diversification will directly lead to the insufficient diversification of assets. Although CoinMarketCap has thousands of different types of coins, but based on the statistics of trading volume on CMC, the transaction of the top 10 coins accounts for up to $85 \%$ of that of all virtual coins, and alt the $85 \%$ transaction is contributed by Genesis Assets. Despite of the large asset quantity and numerous currency types in the current decentralized world, the real asset diversification actually is insufficient.

\section{Closed economic loop without endogenous production}

In the modern economic system, producers borrow money from banks and means of production from the society to produce, and during the process these means of production are consumed to 
make products. The products then be sold by producers to the society in return for capital to pay for the interests on bank loans. Alt these constitute the most primitive closed loop of modern economy. In the closed loop, finance ultimately serves actual production behaviors, which in turn is a process of consuming old products and reorganizing new ones. As long as the earnings from the sale of produced goods are larger than the costs, the wealth in the closed loop will increase accordingly. However, in the current decentralized world, as production and cash-out in the closed loop take place in the real world, the wealth increased during the circulation process does not longer return to the decentralized world. This loop crosses the Gorder between the two worlds, and the interface between the borders is very fragile due to external limitations.

\section{External Limitations}

Since the publication of the white paper for Bitcoin by Satoshi Nakamoto-DBitcoin White Paper: $\mathcal{A}$ Peer-to-Peer Electronic Cash System, the decentralized world of code as law expressed by the blockchain has somehow embodied a free will, a will that knows no borders, no race, no hierarchy. Such will is so unprecedented that bigshots in the world refrain themselves from it out of fear and the majority of people find it beyond their understanding.

This will is concentrated in the distrust of the modern financial system. Satoshi Nakamoto, the pioneer of Glockchains, should have noticed that the modern financial system is likely to come to a dead end in the future and after the collapse of the Bretton Woods System, the modern American dollar system is likely to be weakened after multiple financial crises.

Nonetheless, regardless of what happens in future, such will is incompatible with current patterns and systems.

\section{Exclusiveness of traditional financial system}

Since the invention of joint-stock companies in Netherlands, the modern financial system has nearly 400 years of history. Because of their excessively long history and overly profound accumulation, such financial systems have long lost the capacity to perceive the future at the time of the later-born venture capital operation mode, which makes the already rich richer and serve 
as a tool of the government for economic regulation and control. When virtual assets such as $\mathcal{B} \mathcal{T}^{C}$ tap into the real world from the decentralized world and point to real businesses, it will be very difficult for these financial systems to operate these unprecedented assets in their familiar ways. Therefore, such a financial system will become a huge mountain hindering the decentralized world economy and preventing it from entering into the real world.

\section{The regional nature of political systems}

There are so many countries, systems and national conditions around the globe, and together they constitute the most powerful ecology on the planet - the human society. We aspire to peace and prosperity. However, this ecology is inevitably divided into different economies based on nations or alliances, each with clear and relatively closed boundaries. The decentralized world, on the other hand, is the vast space of freedom that can expand indefinitely beyond these separate economies. Because of the slow pace of regulation cannot keep up with the development of technology, such space will inevitably break the boundaries of economies and disturb the normal functioning of some independent economies. In the decentralized world, grey industries that elude state powers are extremely active. This further aggravates the exclusion of independent economies to decentralized world economy.

\subsection{Break through limitations}

The development of the decentralized world economy will inevitably be faced with the abovementioned limitations. How to break through

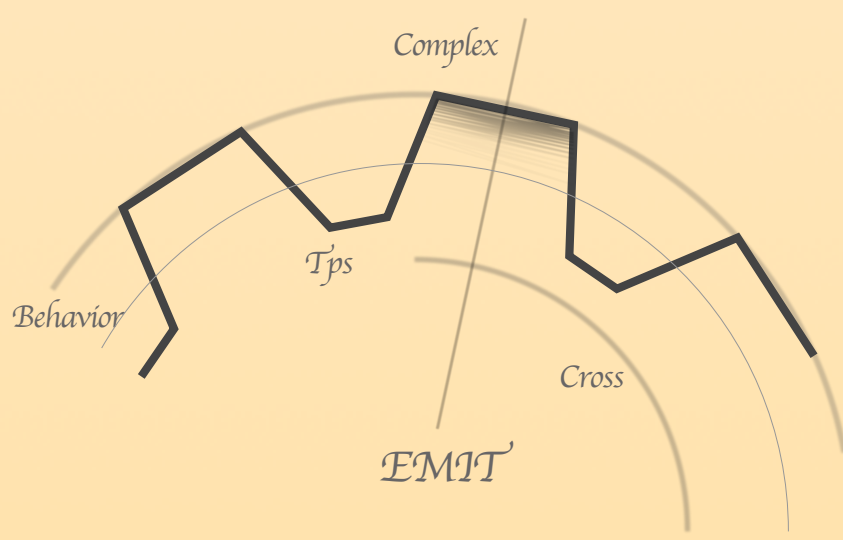
these limitations? $\mathcal{E M I T}$ believes that this mainly depends on two aspects: firstly improving the infrastructure capability and secondly improving the diversification of economic behaviors of the decentralized world. The former is the physical support for the latter, and the latter is a prerequisite to build a decentralized world economy with value support. 
Before introducing $\mathcal{E M} 1 \mathcal{T}$, it is necessary to review what contributions the blockchain industry has made for this purpose intentionally or unintentionally.

\section{A. Infrastructure Upgrades}

\section{T'PS}

The most critical of all is to improve the efficiency of human-computer interaction, that is, T'PS(transactions per second), and the blockchain industry has gone forward and backward with a lot of exploration and experiments since its development. Like $\mathcal{E O S , ~ A l g o r a n d ~ a n d ~} \mathcal{N} \mathcal{E} A \mathcal{R}$ have made a lot of contributions to this matter.

\section{Cross-chain}

In order to link the whole decentralized world, terminate information isolated islands and integrate existing virtual assets, it is necessary to build cross-chain facilities across the decentralized world. $\mathcal{A}$ substantial amount of work has been carried out by virtue of systems including Polkadot, Cosmos and Anyswap to explore this approach.

\section{Complex decentralized applications}

$\mathcal{A}$ reasonably developed decentralized ecosystem should be complex and abundant, and Ethereum's smart contract mechanism is a pioneer in this goal. However, limited by the high costs of storage and computing resources and other problems such as computing complexity, it will be difficult for virtual machines integrated inside native consensus to satisfy the extremely complicated demand for decentralization, and various kinds of loopholes occur easily, so it is not suitable to build an extremely complex ecology. Then, another technological solution is inevitably required to resolve this problem. To achieve this objective, Cosmos and Polkadot have done a lot of explorations, and they resorted to blockchain applications to resolve this problem. However, the development and operation threshold for application sub-chains is not a low barrier.

\section{B. Upgrading of $\mathcal{E}$ conomic Behavior Diversity:}


During the development of the Elockchain industry, compared with infrastructure upgrading, the exploration for roles and behaviors inside the complex economic structures and economies is still at the very early stage. The upgrading of economic complexity mainly depends on the two aspects of asset diversification and behavioral diversity.

The so-called crypto-digital assets with which we have been in contact are fungible like fiat currencies, and their value is reflected in storage, circulation and interest acquisition. For example, the $\mathcal{B} \mathcal{T}^{\prime} \mathcal{C}$ in your wallet has no difference from the $\mathcal{B} \mathcal{T}^{\prime} \mathcal{C}$ in my wallet. If the transaction cost and possible credit risks are not considered, you will definitely not resist exchanging the $\mathcal{B T} C$ with me. If the majority of assets existing in the decentralized world are in this form, their economic structure must be simple and linear. People use them for only three purposes: arbitrage, hedging and payment.

However, during the process of development of Glockchains, another type of asset, namely nonfungible tokens (also referred to as $\mathcal{N} \mathfrak{F} \mathcal{T}$ ' asset), has been overlooked. Released at $\mathcal{E} \mathcal{T} \cdot \mathcal{H}$, Cryptokitty is the first known by the community. All attributes of Cryptokitty are preserved in the blockchains of $\mathcal{E} \mathcal{T} \mathcal{H}$ in the form of unique token (gene). Therefore, each Cryptokitty token has its individual estimate. As N NTT assets have plentiful attributes, your Cryptokitty may have different external characteristics from mine. After the release of Cryptokitty, no project can outshine it in popularity for a very long period.

After being swept through by $\mathcal{D} e \mathcal{F} i$, the decentralized world begins to have other attempts, such as 'ALPACA CIT'Y's alpacas, and W'AX's collectible cards, as well as the more active $\mathcal{N} F \mathcal{T}$ trading marketplace OpenSea. By combining with fungible assets, these $\mathcal{N} F \mathcal{T}$ ' assets bring about some more complicated economic behaviors.

These are all small-scale attempts, or rather these are variations derived from within homogenized assets, without fundamentally changing the purposes of virtual assets: arbitrage, hedging and payment.

\subsection{The Role of $\mathcal{E M I T}$}


The three systems of $\mathcal{E M I T}$ ' have achieved the two above-mentioned methods for breaking through limitations from different levels.

\section{A. EMIT' Core Network}

The "EMIT' Core Network" provided by $\mathcal{E} M \mathcal{M} \mathcal{T}$ ' is a consensus protocol newly created by EMIT' initiators. It has the following characteristics:

- Consensus is naturally sharded and cross-chain.

- Supporting complex asset structures and privacy functions.

- Does not issue native fungible tokens.

- Easy to write complex decentralized applications.

- Has infinitely scalable T'PS.

Therefore, it can flexibly support tremendous access volumes, can build complex and diversified virtual assets and stimulate the economy through $\mathcal{N} F \mathcal{T}$ ' assets and various ecologies, making decentralized applications close to centralized applications in terms of $\mathcal{R} \& \mathcal{D}$ efficiency as well as usage experience.

$\mathcal{E M I T}$ can put the assets of the whole decentralized world on a high quality chessboard, and then this virtual universe can generate infinite changes.

\section{B. EPOCH Ecological Cluster}

$\mathcal{E M I T}$ provides the driving logic, protocols, frameworks and paradigms for "IFPOCH Ecological Cluster", which is centered on the NTFT' assets, driving fungible assets, provides abundant economic behaviors and endogenous economic closure, and reconstructing the economic system of the decentralized world. Driven by $\mathcal{E P O C H}$, a large number of users, investors and developers will participate, forming various independent and directly or indirectly related ecologies with each other. As with any market with a fair system and rich trading freedom, EPOCF will create complex economic structures and provide substantial opportunities for all 
participants in different areas at different times. This ecological cluster can eventually generate a huge GDP (Gross National Product).

\section{GDP Financial Marketplace}

$\mathcal{E M} \mathcal{T}$ ' provides the "GDP Financial Marketplace". As the most crucial decentralized application of $\mathcal{E P O C H}$, which will serve as the hub for the diversified assets and economic behaviors in the EPOCH ecosystem. By virtue of the diversity of $\mathcal{E P O C H}$ ecological cluster, the $G \mathcal{D P}$ financial marketplace can remedy the lack of credit mechanism in the decentralized world and will no longer be limited to simple financial behaviors including arbitrage, risk aversion, investment and excess lending. Similar to the central bank of the decentralized world, GDP financial marketplace will allocate resources to the decentralized world with high efficiency and coordinating conflicts between various ecosystems. 


\section{v. $\mathcal{E M I T}$ 's Plan}

$\mathcal{T}$

he plan of $\mathcal{E M I T}$ ' to achieve its uftimate goal is divided into four phases (major versions), namely "Prelude", "Pioneer", "Kingdom" and "World". Each phase is then divided into several small steps (minor versions). There are still no detailed minor steps for the distant future yet, as the project may have some changes by then, and this Parchment Paper will be updated as $\mathcal{E M I T}$ progresses.

5.1. Prelude (2 Months)

A. $\quad$ World Tree (January 2021)

Purpose: Release the Beta version of cross-chain and recruit super nodes.

\section{B. Birth of Pioneers (February 2021)}

Purpose: Release the cross-chain Release version and recruit the first batch of pioneers entering $\mathcal{E P O C H}$.

5.2. Pioneers (1 Year)

\section{A. Start (March 2021)}

Purpose: Release the first version of the $\mathcal{E P O C H}$ eco-app.

B. Clan

Purpose: Complete the GDP diversified financial marketplace and complete the construction of EMIT' Core Network. 


\section{Ark}

Purpose: Complete the construction of $\mathcal{E P O C H}$ focused ecology on $\mathcal{E M I T}$.

5.3. Nation (3 Years)

Purpose: $\mathcal{E P O C H}$ ecological evolution to form a huge GDP.

\subsection{World (5 Years)}

Purpose: Explore new intellects and enrich decentralized worlds, including but not limited to $\mathcal{A R}, \mathcal{V} \mathcal{R}$, artificial intelligence, brain-computer interface 


\section{VI. $\mathcal{E M I T}$ foundation}

$\mathcal{M} \mathcal{T}$ Foundation is a non-profit organization founded to accept all admissible

$\mathcal{E}$ resources and build $\mathcal{E M} 1 \mathcal{T}$, a large decentralized world, together with other organizations or individuals with the same purpose, ultimately realizing the vision: "Creating wealth with technologies and building complex endogenous economic loop in the decentralized world."

The Foundation's website: https://emit.technology

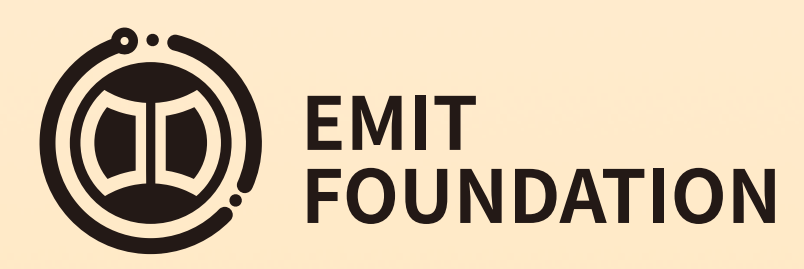




\section{Participation in $\mathcal{E} \mathcal{M} \mathcal{T}$}

articipants prepared to join $\mathcal{E M} \mathcal{M} \mathcal{T}$ may foltow relevant activity arrangements

$\mathcal{P}$ announced by its official media. Starting from February 15 th, all personnel with access to the Internet can participate in the EPOCH ecology. Before that, $\mathcal{E M I T}$ Foundation will organize two launch activities with limited spots starting from January 1st. Participants will be granted with certain powers and obligations and will join the $\mathcal{E M} \mathcal{M T}$ world as the first original inhabitants. These two activities are as follows:

Prospective participants can follow the official $\mathcal{E M I T}$ media related events, starting February 15, where everybody with access to the Internet can participate in the $\mathcal{E P O C H}$ ecosystem. Before that, starting January 1st, the $\mathcal{E} M \mathcal{M} \mathcal{T}$ ' Foundation will organize two kick-off activities, with limited number of people, where participants will get "Limited $\mathcal{E}$ dition Rights" and obligations to join the world of $\mathcal{E M I T}$ first as original inhabitants. These two activities are as follows:

Translated with www.DeepL.com/Translator (free version)

1. World Tree ( $\mathcal{T}_{\mathcal{O}}$ be started on January 1 st)

2. Birth of Pioneers ( $\mathcal{T}_{0}$ be started on January 15 th)

$\mathcal{E M I T}$ can be followed at the following web addresses:

Official Site: https://emit.technology

Official Blog: https://blog.emit.technology

Official Community: https://community.emit.technology

T'legram: https://t.me/EMTI'Protocol

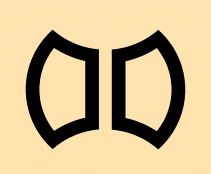

